Document downloaded from:

http://hdl.handle.net/10251/63730

This paper must be cited as:

Navarro Peris, E.; Corberán Salvador, JM.; Ancik, Z. (2015). Evaluation of the potential recovery of compressor heat losses to enhance the efficiency of refrigeration systems by means of thermoelectric generation. Applied Thermal Engineering. 89:755-762. doi:10.1016/j.applthermaleng.2015.06.033.

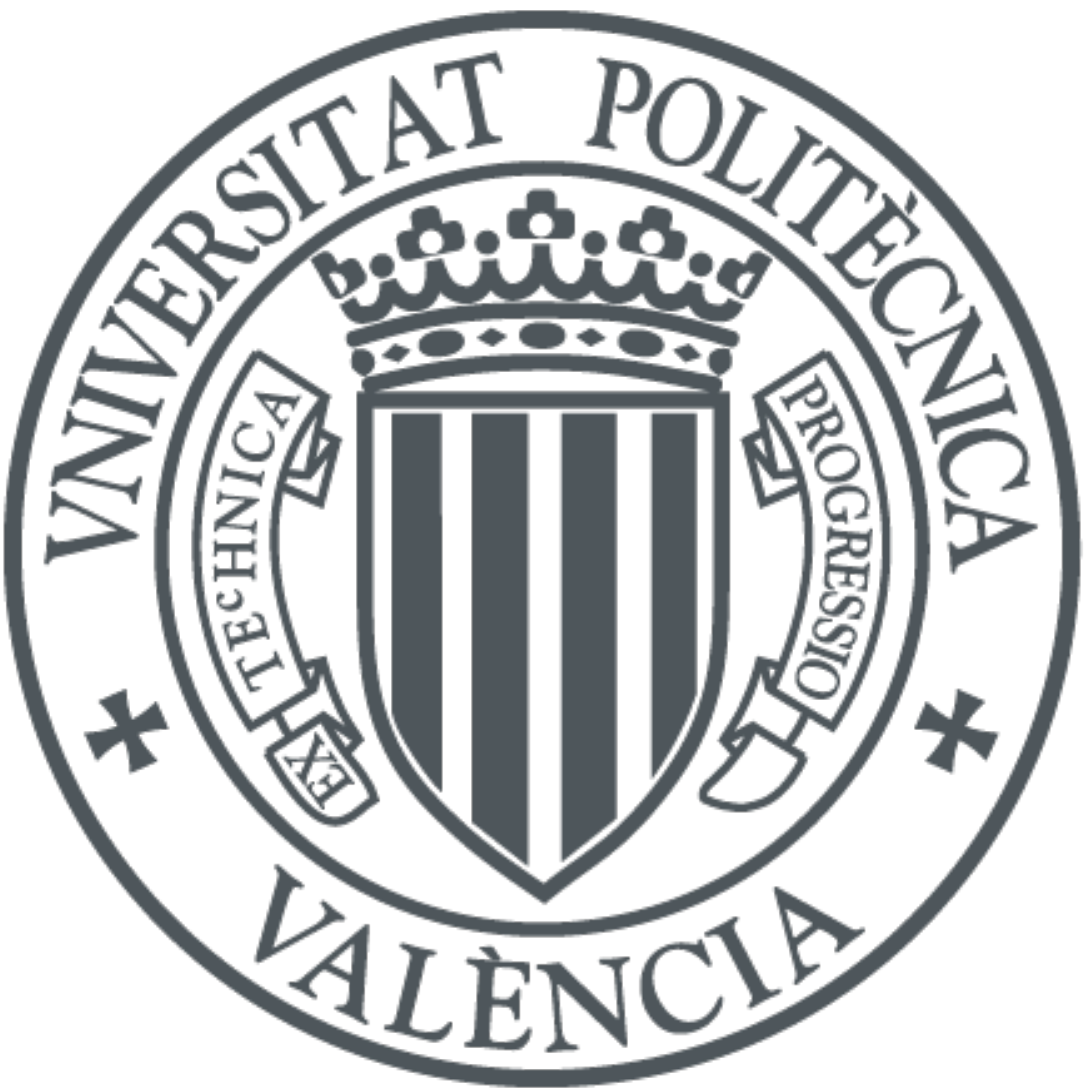

The final publication is available at

http://dx.doi.org/10.1016/j.applthermaleng.2015.06.033

Copyright Elsevier

Additional Information 


\title{
Evaluation of the potential recovery of compressor heat losses to enhance the efficiency of refrigeration systems by means of thermoelectric generation
}

\author{
E. Navarro-Peris*1, JM Corberan ${ }^{1}, \mathrm{Z}$ Ancik $^{2}$ \\ ${ }^{1}$ Instituto de Ingeniería Energética, Universitat Politècnica de València. Camino de Vera \\ s/n, 46022 Valencia, España. Teléfono: 34-963879123, Fax: 34-963877272. \\ ${ }^{2}$ Faculty of Mechanical Engineering, Brno University of Technology, Technicka 2, Brno \\ 616 69, Czech Republic. Tel.: +420 541142 858, Fax: +420 541142876 \\ *Corresponding author: enava@ter.upv.es
}

Keywords: Compressor, Seebeck effect, heat recovery, heat losses.

\begin{abstract}
The present study evaluates the possibilities of increasing the efficiency in refrigeration and heat pump systems based on compression cycles by means of using the compressor heat losses. In order to do that, the work been divided in the following parts: development of a model of a thermoelectric device in order to estimate the amount of energy recovery from the heat source, experimental test under several contour conditions to estimate the real temperature difference maintained in a thermoelectric module in contact with compressor wall and based on this information estimation of the potential amount of energy that can be recovered by this kind of system.
\end{abstract}

Keywords: Thermoelectric effect, Peltier/seebeck effect, harvest, compressor losses

\section{Introduction}

Nowadays there is an increasing interest on reducing the global energy consumption. In that sense, heating and cooling account for a significant proportion of Europe's energy demand and EU is promoting the improvement of the efficiency of that kind of systems.

Vapor compression systems will have a key role in these objectives (more than $90 \%$ of the total systems used) and several alternatives are being applied currently in order to improve the efficiency of these systems and to extend its application range: using more efficient thermodynamical cycles like two stage cycles [1] [2], implementing a inverter in the 
compressor in order to fit the compressor capacity with the system demands, design more efficient compressors, development of improved control algorithms and the like.

On the regular compressor operation, part of the supplied power is released in the form of heat losses to the environment reducing the total efficiency of the system. The amount of energy lost to the environment depends on the working conditions and the size and technology of the compressor. Heat losses of commercial size compressors for heat pumps systems $(3 \mathrm{~kW}-15 \mathrm{~kW})$ can vary from $3 \%$ to $25 \%$ of the total compressor consumption but this losses can increase significantly for compressors of low capacity (50-70\%) [3]. The recovery of part of this energy could lead to an improvement in the global system performance.

Energy harvesting consists of capturing, storing and using energy freely available in the environment [4], [5], [6]. One of prospective ways of energy harvesting is based on ThermoElectric Generator technology (TEG) in which heat is directly converted to electric energy using the thermoelectric properties of some materials and could be applied in any device in which waste heat is rejected to the environment. This is called the thermoelectric effect and is the responsible for creating a voltage in a thermoelectric device when there is a temperature difference between the two sides of the thermoelectric module and conversely, creating a temperature difference across a thermoelectric module when a voltage is applied to it. The intensity of the effect will depend on the employed material and on the applied temperature difference between the hot and cold sides. The selection of the module material critically depends on the operating temperatures and temperature difference of the application. 
The use of the thermoelectric effect for heating and cooling is well known. It has the advantages of being compact, not having moving parts so they can be silent and handy, and have unlimited life. The COP of cooling thermoelectric devices shows a strong dependence on the applied temperature difference. For temperature differences lower than $5 \mathrm{~K}$ the COP can be higher than 10 and this technology could compete with vapour compression systems for small capacities. However, for higher temperature differences the COP of thermoelectric coolers decreases significantly and this kind of technology is not an option if the efficiency is an important factor [7]. Among the current applications of this technology are human cooling/heating, portable cooling/heaters, silent coolers, cooled/heated car seats, cold start for diesel engines, cooling of electronic devices and the like [8], [9].

The use of thermoelectric effect for producing electric energy from heat has low efficiency (values lower than 5\% are typically found). However, there are many processes in which heat is wasted directly to the ambient and recovering $5 \%$ of this energy could be profitable. This fact has made that the use of this effect for energy recovery has been considered in certain applications like small scale electric power sources for electronics in a wide range of applications.

This work analyses the potential of TEGs to enhance the efficiency of a refrigeration system recovering part of the heat losses of the compressor. First, a model for the thermoelectric module was developed. Then, in order determine the validity of the model to evaluate the TEG performance an in order to evaluate the potential for power generation under different cooling conditions, an experiment al test campaign with the system working in real conditions has been carried out. Finally, based on the experimental results the potential of the TEGs to enhance the efficiency of refrigeration systems is discussed. 


\section{Thermoelectric Generator}

TEG is based on the Seebeck effect. The electric connection of two dissimilar conductions or semiconductors creates a thermocouple junction ¡Error! No se encuentra el origen de la referencia.. When different temperatures are applied to both sides of the thermocouple, electric power is generated.

Figure 1. Thermocouple - Seebeck effect ¿Error! No se encuentra el origen de la referencia.

The electric voltage generated by a thermocouple depends linearly on the applied temperature difference.

$$
U=\alpha \cdot\left(T_{H}-T_{C}\right)
$$

Where, $T_{H}$ is the temperature of hot side, $T_{C}$ the temperature of cold side and $U$ the electric voltage. $\alpha$ is the differential Seebeck coefficient which is a material property of the thermocouple junction and it is defined as:

$$
\alpha=\alpha_{p}-\alpha_{n}
$$

Being $\alpha_{p}$ the Seebeck coefficient of material $\mathrm{p}$ and $\alpha_{n}$ Seebeck coefficient of material $\mathrm{n}$.

\subsection{Design of TEG module}


The TEG module is created by thermocouples connected electrically in series and thermally in parallel. Nowadays the three main types of TEGs commercially available are: the common TEG module, the MEMS TEG modules and the Thin-film TEG, which differ in both dimensions and power generation. Thanks to the development and new technologies in the field of material engineering and manufactured processes the performances of TEG modules have significantly improved in the past years [11]. Nevertheless, in this study and for a preliminary analysis in order to determine the potential of this kind of technology, common TEG modules have been used.

Figure 2. Scheme of the TEG design.

Based on the commercially availability, the module described on table 1 was selected:

Table1. Thermoelectric module characteristics

\subsection{Model of the TEG module}

The developed model is divided in a thermal part and an electric part, both connected by eq. (1). Figure 3 represents a scheme of the model.

Figure 3 Scheme of the TEG model. Nomenclature: $\mathrm{R}_{\text {wafer }}$ thermal resistance of wafer, $\mathrm{R}_{\mathrm{tcs}}$ thermal resistance thermoelectric layer, Qjoule heating by Joule effect, QpelH heat flow through the hot side (cause by Seebeck effect), $Q_{\text {pelC }}$ heat flow through the cold side (cause by Seebeck effect), $\Delta T$ temperature difference, $R_{i}$ internal resistance, $R_{L}$ load resistance 
The characteristic properties of the TEG module are calculated according the following equations:

The voltage at the matched load is given by:

$$
U_{L}=\frac{N \alpha\left(T_{H}-T_{C}\right)}{1+2 r \frac{l_{c}}{l}}
$$

where: $N$ is the number of thermocouples, $\alpha$ is the Seebeck coefficient, $T_{H}$ is the temperature of the hot side, $T_{C}$ is the temperature of the cold side, $r$ is the thermal contact parameter, $l_{c}$ is the wafer thickness and $l$ is the thermo element length.

The current through the TEG with matched load is given by:

$$
I=\frac{A \alpha\left(T_{H}-T_{C}\right)}{2 \rho(n+l)\left(1+2 r \frac{l_{c}}{l}\right)}
$$

where: $A$ is the thermocouple area. $\rho$ is the electrical resistivity of the thermocouple materials, $\mathrm{n}$ is the electrical contact parameter and the rest of the parameters have the same meaning as in the previous expression.

Therefore, the power on the matched load results:

$$
P=\frac{\alpha^{2} A N\left(T_{H}-T_{C}\right)^{2}}{2 \rho(n+l)\left(1+2 r \frac{l_{c}}{l}\right)^{2}}
$$

The heat flux through the cold side caused by the Seebeck effect is given by: 


$$
Q_{\text {pelC }}=N T_{C} I \alpha
$$

The heat flux through the hot side caused by the Seebeck effect is given by:

$$
Q_{p e l H}=N T_{H} I \alpha
$$

and the internal heat generation in the TEG results:

$$
Q_{\text {joule }}=R_{i} I^{2}
$$

where $R_{i}$ is the internal resistance

The model is implemented in MATLAB/Simulink and a more detailed description of it can be found in [13].

\subsection{Results of the simulation of TEG generator}

The model of TEG generator is used to simulate the potential module performance. According to expected operational conditions of compressor the temperature of hot side of TEG module is set to $\mathrm{T}_{\mathrm{H}}=100^{\circ} \mathrm{C}$ and the temperature difference across the TEG is varied as a parameter in the range that is expected to have in regular compressor operation. The way in which the temperature of the cold side could be varied in practice will be discussed in the following.

The model input parameters for the used TEG module of table 1 are summarized in table 2, and the obtained results for the obtained voltage current and power production are shown in

figure 5. The figures shows that the voltage and the current increases linearly with the temperature difference for the analysed range, this fact indicates that for the temperature 
variations present in the compressor the selected module do not reach the saturation conditions.

Table 2. TEG module parameters

Figure 5.Model results for the used TEG with an area of $16 \mathrm{~cm}^{2}$

\section{Experimental Campaign}

The model is able to estimate the power production based on the real temperature difference between the two TEG sides, but this temperature difference has to be determined for each application. An experimental test campaign was developed in order to determinate the possible temperature difference that the system is able to maintain in real conditions between the two TEG sides under different possible configurations.

\subsection{Experimental set up}

\subsubsection{Test rig description}

The tests were all carried out in a scroll compressor mounted in the test rig shown in figure 5. The test rig is equipped with several PID control loops (compressor inlet and outlet pressure, superheat and subcooling controls) which allow an adjustment of the refrigerant conditions at compressor inlet (evaporating pressure and superheat) and outlet (condensing pressure) with a precision of $1 \mathrm{kPa}$. The rig is fully automated, and designed to permit working in steady state conditions for long time periods. The test rig will allow fixing several working conditions (evaporating and condensing temperatures) in order to change the compressor temperature.

The TEG was placed at the top of the scroll compressor. This situation was selected because there is where a higher temperature is found for this compressor design, as it is shown clearly 
in figure $9 \mathrm{~b}$. The maximum temperatures available at the compressor head depend on the operating conditions, but $90{ }^{\circ} \mathrm{C}$ is typical for many heat pump applications.

A good contact between the TEG and the compressor shell is critical in order to obtain the maximum energy recovery. The TEG module was adapted to the head of the compressor by means of an aluminium piece which was specifically manufactured in order to fit the best as possible the compressor cap, this point is critical in order to maximize the energy recovery, as a consequence of that, additionally, a conductive paste was used to minimise the thermal resistance between the aluminium piece and the head of the compressor. The ensemble was then clamped to the compressor head in order to minimize the thermal resistance between each pair of components.

Figure 6 shows the installation of the TEG module. The piece in blue is made of insulation material and it is inserted in order to decrease the heat transfer from the module, heat sink and aluminium adapting-piece, to the environment.

Three calibrated thermocouples were installed to measure the temperature of the compressor cap, the temperature at the bottom of the TEG module and the temperature at the top of the module, respectively.

In order to be able to measure the power produced by the TEG module an electrical resistance must be placed as a load. The value of this thermal resistance affects the voltage and therefore the module performance. This kind of circuit has an optimum voltage value for the power production. Calculations of the electrical resistance required for this application were made with a simple electrical model for the different planned temperature differences. It turned out that an average value of $2.7 \mathrm{Ohm}$ was sufficiently close all the optimum values. Therefore, the tests were all done with a load resistance of that value.

Power was directly evaluated from current and voltage measurements on the load resistance. 
Figure 5. Experimental test rig used for the test.

\subsection{Performed experiments}

As it is commented previously, TEG module electric power is directly related to the temperature difference between the two sides of the module. When the module is placed at the top of the compressor shell, although it can be possible to have a temperature difference of $80^{\circ} \mathrm{C}$ between the compressor cap and the environment, the temperature difference between both sides of the module is significantly smaller because of the heat transfer by

conduction through both TEG sides and by the limited heat transfer between the module and the ambient as a consequence of the low natural convection coefficient.

In order to increase the temperature difference between the two module faces several alternatives have been evaluated in this work:

I. Heat sink on the top of the TEG module with natural convection over the heat sink

II. Heat sink on the top of the TEG module with forced convection over the heat sink.

III. Water heat exchanger on the top of the TEG module with forced convection in the water chamber.

IV. Refrigerant heat exchanger on the top of TEG module with small refrigerant liquid injection. The heat exchanger is physically the same that the one employed for case III with water.

Figure 6. Three TEG configurations tested. 
Figure 7 shows a photograph of the TEG module, the heat sink, the aluminium adapting piece and the heat exchanger.

A remark should be made here, the objective of this test campaign was to be able to impose several temperature differences between both sides of the TEG, but not to propose a methodology to obtain that temperature in a real system. Therefore, the use of a fan or water pump in order to cool down one of the sides is justified for these test, but not in a real system in which if the electric requirements of the fan has to be included on the energy balance the net production of energy by the TEG would be close to 0 or even negative. The point that can constitute a real alternative for the refrigeration of this kind of system would be for instance the use of the refrigerant from the outlet of the evaporator or other possible cooling sources without any additional energy cost.

Figure 7. Image of the thermoelectric module mounted at the top of the compressor and coupled to the water heat exchanger.

\section{Results and discussion}

Changing the compressor working conditions and using the different configurations described on the previous section the power obtained by the TEG generator was measured and the results are shown in figure 8.

Figure 8 Produced power produced by the TEG as a function of temperature difference.

The figure shows that enhance the heat transfer only with a heat sink and natural convection is not able to produce a temperature difference higher than $30 \mathrm{~K}$ on the device. Adding a fan 
to the heat sink allows the increase of the temperature up to $45 \mathrm{~K}$ but the energy of the fan should be added to the total energy consumption of the system. The system maintaining a higher temperature between both sides of the TEG module uses a liquid cooler, in that case the temperature difference could reach values up to seventy degrees in the more extreme conditions resulting in a higher energy recovery potential. It was not possible to increase more the temperature difference between both sides with the selected module because at this temperature difference limit the hot side of the system begins to cool down.

Regarding the efficiency of the conversion rate it has been between $1.5 \%-1 \%$. This value and according the nominal specifications of the module points to the fact that the used thermoelectric module is working in a temperature range which is not closer enough to its optimum value. Therefore using a more adapted to the temperature range module could increase the amount of energy recovered.

These experimental results were also used in order to validate the developed model in such a way that it can assist in the design of future prototypes. Table 3 shows the results obtained in the model validation.

Table 3. Comparison of open voltage measured and open voltage simulated by the thermoelectric generator for different temperatures of the hot and the cold side. The first row corresponds to the reference value to which all the module parameters are defined.

The model is able to predict the electric potential generated in nominal conditions but some deviations have been observed for the rest of the cases. The most influent factor on the deviation is the dependence of the Seebeck coefficient on temperature that has not been taken into account in this model because there was no information about that. Nevertheless, the authors consider that the results supplied by the model can be considered as a good estimation 
of the actual power production in order to extrapolate these results to other working conditions.

The used compressor for these experiments was a scroll compressor with $5.35 \mathrm{~kW}$ of nominal electrical power in the conditions Tevap $=-20$, Tcond=45. In these conditions, the maximum temperature of the compressor shell was $90^{\circ} \mathrm{C}$. The external surface that the compressor has is approximately $0.05 \mathrm{~m}^{2}$. It can be established that $1 / 5$ of the surface of the scroll compressor is at these temperature conditions (see figure $9 \mathrm{~b}$, where the thermography of the scroll compressor is shown). Therefore, assuming that all the surface at high temperature could be covered by thermoelectric material of these characteristics, the maximum power at these conditions that the thermoelectric generator could recover from this compressor would be from $15 \mathrm{~W}$ to $60 \mathrm{~W}$ depending on the selected method to cool down the cold side of the thermoelectric material. In principle this power usually has been used to feed some microelectronic circuits of the system, but in refrigeration applications as the system perform cyclic operations of start and stop it is difficult to use this energy directly and it should be stored losing part of the global efficiency.

Another energy recovery possibility for this kind of systems would be to use this energy to feed up a TE device in order to produce heating or cooling. As it has been commented on the introduction, TE modules for heating and cooling have the property of having a high COP for small temperature differences. If we have an air condenser properly designed, the temperature difference between the refrigerant at the outlet of the condenser and the air of the environment should be small. In this cases coupling a TEG module on this part of the system increases the subcooling which will rise the cooling capacity of the system without any additional cost. This alternative has been evaluated by some authors in the past 
[1],[14],[15] and particularly for this special application the following advantages can be cited:

a) The increase of cooling capacity of the system only is required when the system is working, therefore, the energy is used as it is recovered from the compressor and no energy storage system is required.

b) The cooling demand is higher when the cooling temperature is lower and when the ambient temperature is higher, these conditions usually means high compressor ratios. High pressure ratios in the compressor have higher heat losses and higher compressor temperatures. These are the best conditions to recover compressor energy using TEG.

c) The TEG system could help to couple the system cooling demand to the system production capacity increasing the global efficiency of the system.

In order to quantify this approach, the potential subcooling obtained with this system using the thermoelectric device has to be estimated. Assuming that the COP of TEG systems is given by the expression [16]:

$C O P=Q_{c} / E=\frac{\alpha I T_{c}-k \Delta T-0.5 I^{2} R}{I^{2} R+\alpha I \Delta T}$

Where $\mathrm{Tc}$ is the temperature of the cold side and $\Delta \mathrm{T}$ is the temperature difference between the cold and the hot side.

Figure 9. Thermography of: a) Piston compressor, b) Scroll compressor c) Rotary compressor 
Based on the experimental values obtained for the TEG electric generation (see figure 8), extrapolating them for a more precise system in which the top of the compressor shell is covered by TE material (an area of approx. $0.05 \mathrm{~m}^{2}$ ) and considering that all recovered energy can be sent to a cooling system based on thermoelectric phenomena at the outlet of the condenser with a cooling capacity assuming a the COP given by expression (9), figure 10 represents the estimation of the cooling capacity that can be recovered/produced by this kind of system for this type of compressor technology according the data obtained for the TEG configuration with a water heat exchanger (configuration c)). Figure 10 shows that more than $500 \mathrm{~W}$ can be reintroduced in the system by this kind of approach.

Figure 10. Cooling capacity produced by the thermoelectric system from the power generated in the TEG with the water heat exchanger configuration.

Figure 9 represents a thermography of a piston compressor, a scroll compressor and a rotary compressor. The figure shows that the lowest temperatures of the compressor shell are found for the piston compressor and the highest temperatures are found in the rotary compressors and scroll compressor. The percentage of the compressor shell area at high temperature can be increased if this system is applied to rotary compressors in which almost all the shell is at high temperature having more heat losses. In this type of compressor design, the energy recovery could be more effective.

Finally, it should be commented that when the system is built with a TEG covering the compressor shell a temperature reduction in the shell is expected. This temperature reduction probably will imply a reduction of the energy production by the system. A deeper analysis 
of this effect from the experimental point of view would be required in order to be able to estimate it with a significant precision, however, from the point of view of compressor reliability and efficiency this effect will also contribute positively to the compressor performance in extreme conditions reducing its shell temperature.

\section{Conclusions}

Thermoelectric generator has been tested under several conditions varying the temperature difference between both sides of the thermoelectric module. The results have shown that the temperature difference maintained by natural convection maintain between both sides of the TEG is very small and the energy produced from this system kind of configuration will be quite low.

In order to be able to maintain a significant temperature difference between the two faces of the TEG another kind of cooling methods should be used. Three additional strategies have been analysed in the paper: Forced convection, water heat exchanger and refrigerant evaporator heat exchanger. According the obtained results, methodologies requiring additional input energy like forced convection are not feasible and only the methodologies based on a refrigerant or water flow have shown potential to be applied in real systems. The temperature difference obtained with these technologies have been demonstrated as effective and a higher efficiency cooling down the cold side of the TEG will not be produce a higher temperature difference with the used module (heat transfer by conduction will reduce the total temperature difference). From the practical point of view, the use of part of the refrigerant flow coming from the outlet of the evaporator would be a feasible solution in order to maintain the temperature difference between the two faces of the TEG module. 
From all the performed experiments, recovery up to $1.5 \%$ of the total compressor losses has been demonstrated feasible. This amount could be increased by a better selection of the thermoelectric material for the working temperatures.

The efficient use of the recovered energy is not easy in this kind of system (energy production rate low, system starts and stops...) but considering that thermoelectric devices have high COPs when they are working as cooling devices for low temperature differences between the hot and the cold side and that TEG is recovering the heat losses of a cooling machine, the possibility of reusing the electric generation in a thermoelectric cooling device has been analysed.

Considering the characteristics of the thermoelectric material and for the scroll compressor used in these test, it has been shown that in this kind of configuration, it is possible to increase the cooling capacity in $3.5 \%$ without an increase in the electric demand. It is noticeable that this configuration have a higher increase in the cooling capacity when the pressure ratio in the compressor increases which correspond with higher compressor losses and higher reduction in cooling capacity of the system.

The potential of this methodology to recover compressor losses can be increased if other compressor designs with the shell at higher temperatures like rotary compressors are used.

In the development of the experiments, it has been critical to have a good contact between the compressor and the thermoelectric module in order to obtain a significant energy recovery. For that reason a piece of aluminium has been built in order to adapt the thermoelectric material to the compressor cap. Therefore, in order to obtain the reach the potential capabilities of this kind of systems in a real machine, the adaptation of the 
thermoelectric material to the compressor and to the condenser outlet must be obtained. The compressor part should be designed by compressor manufacturers and for the pipe at the outlet of the condenser, cylindrical systems like the ones used by the car industry [17] in order to recover energy from the engine exhaust gasses, adapted to the temperature ranges involved in this case, could be good candidates to bring this approach to reality. 


\section{References}

[1] R Rademacher B Yang. Integrating alternative and conventional Cooling technologies. ASRHAE JOURNAL. October. 2007.

[2] M Akei K Ignatiev, N Jayanth, H Pham, J Caillat, Vapor compression circuit and method including a thermoelectric device. US patent 7296416 B2. 2007.

[3] Ribas FA, Deschamps CJ, Fagotti F, Morriesen A, Dutra T, Thermal Analysis of Reciprocating Compressors -A Critical Review. International compressor engineering conference (Purdue). (2008).

[4] Priya S., Inman D. J. "Energy Harvesting“, Springer 2009, ISBN 978-0-387-764641.

[5] Hadas, Z.; Singule, V., "Energy harvesting - opportunity for future remote applications," 17th International Conference on Engineering Mechanics, Svratka, Czech Republic, 167-170 (2011).

[6] Hadas Z., Singule V., Vechet S., Ondrusek C., "Development of energy harvesting sources for remote applications as mechatronic systems." IEEE 14th International Power Electronics and Motion Control Conference 2010, Ohrid, Macedonia, 13-19 (2010).

[7] Astrain D, Vian JG, Dominguez M. Increase of COP in the thermoelectric refrigeration by the optimization of heat dissipation. Applied Thermal Engineering, 23, 2183-2200, (2003).

[8] Riffat SB, Ma X, Thermoelectrics: a review of present and potential applications. Applied Thermal Engineering, 23, 913-935, (2003).

[9] Bell L. Cooling, Heating, Generating Power, and Recovering Waste Heat with Thermoelectric Systems. Science, 321, 1457-1461, (2008).

[10] Rowe D.M. at al., "Thermoelectrics handbook : macro to nano", Taylor \& Francis Group, ISBN 0-8493-2264-8, (2006).

[11] Goldsmid H. J., "Introduction to Thermoelectricity", Springer Heidelberg Dordrecht London NewYork, 0933-033 (2010).

[12]Z. Ancik; R. Vlach; L. Janak; P. Kopecek; Z. Hadas, "Modeling, simulation and experimental testing of the MEMS thermoelectric generators in wide range of operational conditions". Proceedings of SPIE Vol. 8763, 87631M (2013)

[13]Janak L., "Application of MEMS technology in the field of thermoelectric generators", BUT Brno, (2012)

[14] Schoenfeld JM, Integration of a thermoelectric subcooler into a carbon dioxide transcritical vapour compression cycle refrigeration system. Master Thesis, University of Maryland. (2008)

[15] Sarkar J. Performance Optimization of a transcritical CO2 refrigeration cycle with thermoelectric subcooler. International Journal of Energy Research, 37, 121-128. (2013).

[16] Riffat SB, Ma X. Improving the coefficient of performance of thermoelectric cooling systems: a review. International Journal of Energy Research. Vol 28, 753-768 (2004).

[17] Thermoelectric waste heat recovery program for passenger vehicles. 2013 DOE Hydrogen and Fuel Cells Program and Vehicle Technologies Program Annual Merit 
Review and Peer Meeting. http://energy.gov/eere/vehicles/downloads/thermoelectric-waste-heat-recoveryprogram-passenger-vehicles-0 (last visit January 2015). 


\section{Figures}

Figure 1. Thermocouple - Seebeck effect ¡Error! No se encuentra el origen de la referencia.

Figure 2. Scheme of the TEG design.

Figure 3. Scheme of the TEG model. Nomenclature: $\mathrm{R}_{\text {wafer }}$ thermal resistance of wafer, $\mathrm{R}_{\mathrm{tcs}}$ thermal resistance thermoelectric layer, $Q_{\text {joule }} \quad$ heating by Joule effect, $Q_{\text {pelH }}$ heat flow through the hot side (cause by Seebeck effect), Qpelc heat flow through the cold side (cause by Seebeck effect), $\Delta T$ temperature difference, $R_{i}$ internal resistance, $R_{L}$ load resistance

Figure 4.Model results for the used TEG with an area of $16 \mathrm{~cm}^{2}$

Figure 5. Calorimeter test rig.

Figure 6. Three TEG configurations tested: $1^{\mathrm{o}}$ adaptor to the compressor cap (in grey) isolation material, TEG module (in green), disipartor (orange). $2^{\circ}$ adaptor to the compressor cap (in grey) isolation material, TEG module (in green), disipartor (orange), fan. $3^{\circ}$ adaptor to the compressor cap (in grey) isolation material, TEG module (in green), water heat exchanger (in orange).

Figure 7. Image of the thermoelectric module mounted at the top of the compressor and coupled to the water heat exchanger.

Figure 8 Produced power produced by the TEG as a function of temperature difference.

Figure 9. Thermography of: a) Piston compressor, b) Scroll compressor c) Rotary compressor

Figure 10. Cooling capacity produced by the thermoelectric system from the power generated in the TEG with the water heat exchanger configuration. 
